\title{
EL CUIDADOR FAMILIAR. UNA REVISIÓN SOBRE LA NECESIDAD DEL CUIDADO DOMÉSTICO Y SUS REPERCUSIONES EN LA FAMILIA
}

\author{
Pilar Isla Pera \\ Departament d'Infermeria de Salut Püblica, Salut Mental i Maternoinfantil \\ Escola Universitària d'Infermeria, Campus de Bellvitge. L'Hospitalet de Llobregat
}

\section{RESUMEN}

En los últimos 50 años se han producido importantes cambios en el patrón epidemiológico. El aumento de la esperanza de vida y el descenso de la natalidad han originado un envejecimiento de la población y una mayor prevalencia de enfermedades crónicas. Aunque el envejecimiento es un fenómeno universal, en España, se está produciendo a una velocidad mayor que en otros países. Se espera que la población mayor de 80 años aumente su tamaño en un $66 \%$ entre 1986 y 2010. La mayor parte de los cuidados que necesitan las personas con enfermedades crónicas o invalidez, se dispensan en el núcleo familiar y aunque desde la década de los 80 se observa una mayor preocupación por los cuidadores informales no existe un sistema de apoyo institucional eficaz. A través de una revisión bibliográfica de los últimos 5 años, se analiza la situación actual en nuestro país y se reflexiona sobre el futuro de estos cuidados, debido a los cambios profundos que se están produciendo en las formas de vida de la familia. También se hace hincapié en las repercusiones físicas, psicológicas y sociales que tiene el hecho de cuidar para el cuidador principal y sobre el proceso de atención a la familia que deberían desarrollar los profesionales, especialmente la enfermera.

\section{SUMMARY}

In the last 50 years important changes have taken place in the epidemiological pattern. The life expectancy increase and the birth rate decrease have caused an ageing in the population and a higher prevalence of chronical diseases. Although this ageing is a universal phenomenon, in Spain it is faster than in other countries. It is expected that population over 80 will increase by $66 \%$ between 1986 and 2010. Most of the care required by people with chronical diseases or disabilities are provided within the core family and, although since the 80's a higher concern for caregivers has emerged, there is no effective institutional support system yet. Through a bibliographic revision of the last 5 years, the current situation in our country is analized and the future of this caretaking is thought over, due to the deep family lifestyle changes that are taking place. The physical, psycological and social implications of caretaking on the main caregiver are emphasized, as well as the process of family assistance that should be developed by medical professionals, and above all, by the nurse.

El cuidado informal se puede definir como aquella prestación de cuidados a personas dependientes por parte de familiares, amigos y otras personas que no reciben retribución económica por la ayuda que ofrecen. Numerosos estudios demuestras que son los miembros de la familia los que con mayor frecuencia prestan este tipo de cuidado'. 
El patrón epidemiológico ha sufrido importantes cambios en los últimos 50 años, el descenso de la natalidad y el aumento de la esperanza de vida conllevan un envejecimiento de la población y una mayor prevalencia de enfermedades crónicas. Los avances científicos permiten que muchas personas con enfermedades congénitas, accidentes y otros procesos morbosos graves, puedan sobrevivir, pero muchas veces quedan con algún tipo de incapacidad residual y necesitan cuidados permanentemente. La realidad ès que sólo una mínima parte de la atención en salud está asegurada por los servicios sanitarios, ya que la mayoría de los cuidados son autodispensados o suministrados por la familia². M.A. Duran afirmaba en 1991, que mientras el sistema sanitario formal dispensa un $12 \%$ del tiempo total dedicado a la salud, el $88 \%$ restante forma parte del trabajo realizado en el ámbito doméstico ${ }^{3}$.

Aunque el envejecimiento de las poblaciones humanes es un fenómeno universal, en España los cambios demográficos están ocurriendo a una velocidad mayor que en otros países desarrollados debido a la extraordinaria disminución en la fertilidad y al aumento de la esperanza de vida. Si bien en todos los países, la población mayor de 80 años es el grupo demográfico con mayor crecimiento, en España se espera que este grupo aumente en un $66 \%$ su tamaño entre 1986 y 2010 , superando el millón y medio de personas ${ }^{4}$. En ausencia de una oferta de servicios sociosanitarios, la familia asume la práctica totalidad de los cuidados de los mayores dependientes. La cobertura de los programas públicos de atención a domicilio es inferior al $1 \%$ de la población mayor y la oferta pública de instituciones es también muy escasa, con excepción de Catalunya ${ }^{5}$.

Según el informe SESPAS 6 , en 1997, el 14\% de la población española tenía más de 65 años. La fertilidad en España había descendido a 1,2 hijos por mujer, muy lejos del 2,1 necesaria para el rem- plazamiento. Por el contrario la esperanza de vida al nacer se situaba en máximos históricos: 81 años para la mujer y 74 para los hombres. En España 4 de cada 5 defunciones se producen después de los 65 años y el 44\% de las defunciones se producen después de los 80 años. Esto genera que las principales causas de defunción sean las enfermedades crónicas y como consecuencia de estos cambios en los patrones de la morbilidad existe un aumento de incapacidades funcionales que demandan cuidados de larga duración. Con relación a los cambios de salud asociados al envejecimiento poblacional hay que destacar el problema de las enfermedades neurodegenerativas. Entre estas, cobran gran importancia las demencias, por la magnitud del problema actual y por el aumento esperado debido al incremento de personas mayores de 85 años ya que la incidencia de demencias aumenta con la edad. Diversos estudios estiman una prevalencia del 2,4\% en el grupo de 65-74 años; del 11,1\% en el grupo de 75-84 años y del 34,5\% en mayores de 85 años. Según estas estimaciones España tiene entre 450.000 y 500.000 casos de demencia y casi la totalidad de estas personas vive en la comunidad.

Un estudio realizado en Madrid demostró que sólo 1 de cada 20 pacientes diagnosticados se encontraba en una residencia ${ }^{7}$. Esta situación lleva a plantear el problema del cuidador familiar por las repercusiones que tiene: Un estudio realizado en 1997 demostró que la depresión es una afectación frecuente en los cuidadores de familiares con pérdida de autonomía y confirmó que la atención de los servicios sociales públicos a estas familias es prácticamente inexistente. Las fuentes de apoyo externas a la familia suelen ser de pago privado ${ }^{8}$.

La discapacidad es también atendida, en un 90\% de los casos, en el ámbito doméstico. Y finalmente, los niños constituyen un grupo específico en cuanto a la demanda de cuidados de salud, debido a las características de mayor dependencia, 
no sólo por las enfermedades crónicas o discapacidades sino también por los procesos agudos leves que ocasionan una sobrecarga importante de cuidados en el ámbito familiar'.

Si bien la familia siempre ha sido cuidadora de sus miembros, es a partir de los 80 cuando aparece la preocupación por "los cuidadores". Ello es debido a diferentes factores: 1) El crecimiento económico de los años 60 decrece y a finales de los 70 se inicia el declive de las economías de los países occidentales con la consiguiente preocupación por la utilización eficiente de los recursos. Ello hace que se comience a cuestionar "el estado del bienestar"; 2) Los cambios demográficos, sobre todo el envejecimiento de la población, ya mencionado. 3) El reconocimiento de la magnitud e importancia del cuidado informal y una mayor comprensión del grado en que todavía son operativos los antiguos modelos de la relación familiar, aunque paralelamente también nace la preocupación por la creciente incorporación de la mujer en el mercado laboral y el aumento de los divorcios; 4) La reforma de los sistemas sanitarios que centran su interés en la Atención Primaria de Salud cuyos objetivos son proporcionar una atención integral y continuada a la comunidad lo que conlleva un aumento del interés por la atención comunitaria, por mantener a las personas dependientes en su ámbito, dentro de la comunidad y 5) Se genera una protesta del movimiento feminista puesto que la atención comunitaria la proporciona la mujer y se comienzan a exponer las cargas diferenciadas soportadas por las mujeres.

Aunque son miembros de la familia los que ejercen la función de cuidar, suele ser una sola persona la que asume la mayor parte de la responsabilidad y por lo tanto soporta la mayor sobrecarga física y emocional del cuidado del paciente. A esta persona, que generalmente es una mujer, se la denomina "cuidador principal".
En un estudio sobre solidaridad familiar y vejez $^{10}$ (Bazo y Dominguez-Alcon) realizado, en 1995, en el País Vasco, Comunidad de Madrid y Catalunya, y centrado en los cuidados de salud en la familia para las personas ancianas, se obtuvieron unos resultados que confirman la hipótesis de que la familia es la principal proveedora de cuidados y que en el grupo familiar las mujeres asumen mayoritariamente esa responsabilidad. Las cuidadoras son esposas e hijas. La proporción de ambas en el País Vasco y Madrid es similar mientras que en Catalunya es algo más elevada la proporción de hijas. La participación de nueras es más reducida. Existen varones cuidadores pero su presencia es testimonial, generalmente son maridos que cuidan a sus esposas o hijos solteros que cuidan a sus padres. La edad de los varones que cuidan es elevada, entre 60 y 70 años o más. La edad de las mujeres es variable: En Catalunya la edad oscila entre los 55 y 76 años, con excepción de un pequeño grupo de mujeres más jóvenes. Por el contrario, en Madrid existen dos grupos de cuidadoras bien diferenciados: Uno entre los 37 y 50 años y otro de cuidadoras mayores, entre los $70 \mathrm{y}$ los 80 años.

Las diferencias de edad entre las cuidadoras están en relación con el parentesco de la persona a la que cuidan. En Madrid el grupo más numeroso es el de esposas mientras que en Catalunya y el País Vasco es el de hijas.

Según resultados de este estudio la atención sanitaria que ofrece la cuidadora tiene unas connotaciones que merecen destacarse ${ }^{10}$ :

1) Garantiza la atención completa de la persona cuidada ("hacérselo todo").

2) La atención es continuada, se siente responsable en todo momento y no puede dejar de atender a la persona dependiente.

3) Demuestra la solidaridad familiar expresando el deseo de mantener a las personas dependientes en el hogar. 
4) Generalmente el cuidado se expresa como un deber, se sienten con la obligación de hacerlo. Suele estar presente la manifestación de reciprocidad, el sentimiento de no defraudar las expectativas de la persona que recibe los cuidados.

5) Los sentimientos que expresa el cuidador en relación a la persona cuidada tienen diferentes matices, generalmente son gratificantes pero algunas veces son complejos, expresan resignación e impotencia, soledad, desesperación. A veces existen quejas sobre comportamientos poco amables por parte de la persona cuidada, aunque se intentan justificar.

6) A menudo expresan quejas sobre las repercusiones que el cuidar ha tenido en la vida laboral de la mujer y también con la restricción de libertad individual y familiar. Se constata un empobrecimiento de las relaciones de las cuidadoras con el entorno.

7) Las personas cuidadoras mencionan el cansancio físico, el agotamiento, el desánimo, trastornos del descanso y sueño. En ocasiones las repercusiones son más severas y ocasionan depresiones e incapacidad intensa para asumir la situación.

8) También es constante la expresión de quejas por la falta de ayuda social. Se constata un desconocimiento grande sobre las escasas ayudas disponibles así como la convicción de la dificultad de obtenerlas.

9) La cuidadora concibe su papel como parte de sus responsabilidades familiares.

El hecho de cuidar en el domicilio repercute sobre la salud familiar y especialmente sobre la salud del cuidador principal y por ello repercute sobre el funcionamiento de la familia. Diferentes estudios han mostrado que el cuidador dedica mucho tiempo al cuidado y ello representa una sobrecarga física y emocional que altera su vida ${ }^{1-12-13}$.

Algunos autores denominan a estas alteraciones "El sindrome del cuidador ${ }^{\text {14.15. }}$. La ansiedad del cuidador es mayor cuando cuida a un familiar de primer grado $\mathrm{y}$ con frecuencia se siente atrapado emocionalmente, hasta llegar a sentirse culpable cuando piensa en sí mismo'7. Aunque la mayoría de estudios hacen referencia al cuidado de personas ancianas dependientes también existen estudios que corfirman las repercusiones físicas, psicológica y sociales del cuidador de enfermos cróni$\cos ^{18-10-20-21-22-23}$.

Los cambios en las formas de vida de la familia crean algunos interrogantes sobre el futuro de los cuidados que se han de prestar a las personas dependientes en la comunidad. ¿Cuáles son y cuales deberían ser las interrelaciones entre los sistemas sanitarios y sociales y el sistema informal de cuidados?. Es evidente que un elemento central de este debate se deberá centrar en las estrategias de apoyo al sistema familiar.

La familia es una estructura compleja y articulada, presente en todos los sistemas sociales conocidos $^{24}$. A la familia se la ha definido de múltiples maneras y desde diferentes perspectivas, atendiendo a criterios de consanguinidad, de relación legal, de convivencia, de lazos emocionales etc. ${ }^{25}$. La evolución que ha sufrido la familia y el significado que esta tiene para las diferentes culturas ha permitido una amplia variedad de definiciones, no obstante es necesario ser flexible y acoger dentro del concepto de familia no sólo aquellos que comparten el mismo apellido o lazos de sangre, sino también a los grupos que comparten el sentimiento de "sentirse familiaro". De esta manera se puede definir la familia como un grupo de personas, relacionadas entre sí, biológica, legal o emocionalmente, que no necesariamente conviven en el mismo hogar, y que comparten una historia común y unas reglas, costumbres y creencias básicas, en relación con distintos aspectos de la vida en general, y con el cuidado de la salud en particular. En este grupo tienen lugar acciones de autocuidado y de cuidado a personas dependientes llevadas a cabo por los distintos componentes del mismo ${ }^{25}$. 
Leininger ${ }^{27}$ define la práctica del cuidar como un fenómeno de asistencia, apoyo o facilitación a otro individuo con necesidades anticipadas o evidentes, con el objetivo de mejorar la condición humana o del estilo de vida. La autora afirma que existe una diversidad del significado de los patrones, valores o símbolos que envuelven el cuidado, representaciones presentes en la esfera cultural que determinan las acciones y decisiones relacionadas con el cuidado.

Collière $^{28}$ afirma que el cuidar es un acto indispensable no sólo para la vida de los individuos sino para la permanencia del grupo social.

El autocuidado se puede definir como el conjunto de acciones intencionadas que la persona realiza para sí misma o para otros que dependen de ella, con el objetivo de mantener su vida, su salud o su bienestar ${ }^{25}$. Está fuertemente influenciado por la cultura del grupo de referencia donde se desarrolla el individuo. Son conductas aprendidas.

Pearsons y Bales ${ }^{29}$ (1955) definen el "sistema relacional" como un conjunto constituido por dos o más unidades vinculadas entre sí de modo que el cambio de estado de una unidad va seguido por un zambio en las otras unidades; este va seguido de nuevo por un cambio de estado en la unidad primitivamente modificada, y así sucesivamente. De acuerdo con esta definición la familia es un sistema relacional abierto en tanto que esta formada por un zonjunto organizado de elementos (sus miembros) que se relacionan e interactúan entre sí de tal nanera que cualquier acción, alteración o cambio sn uno de ellos repercute en todos los demás y riceversa.

La familia desempeña un importante papel :n el desarrollo integral de todos sus miembros, no ;ólo por el cuidado a las personas dependientes ;ino porque también es un núcleo generador de rábitos; la familia es el entorno en el que los seres uumanos aprenden e interiorizan las bases del utocuidado y del cuidado a personas dependien- tes. De alguna manera la salud de las familias determina la salud de la comunidad.

Sin embargo a pesar de que conceptualmente ya no se discute la importancia de la atención a la familia y que está contemplada en la reforma de la atención primaria, lo cierto es que todavía hoy constituye, en los profesionales sanitarios, una asignatura pendiente, aunque se constata la preocupación creciente por el tema ${ }^{30-31-32-33-34-35-36}$.

Alfaro $^{37}$ define el proceso de "atención de enfermería" como el conjunto de acciones intencionadas que la enfermera realiza en un orden específico, con el fin de asegurar que una persona o grupo de personas necesitadas de cuidados de salud reciban el mejor cuidado posible de los profesionales de enfermería. El proceso de atención a la familia y a la comunidad ha de ser el mismo que se aplica cuando se dirige la atención a un individuo. En ambos casos se ha de partir de un modelo conceptual que guíe el proceso de atención en sus diferentes etapas. Utilizar la teoría general de sistemas permite al profesional de enfermería observar al individuo, familia y comunidad en sus interacciones entre ellos y entre su ambiente. Una de las ventajas es que tiene en cuenta y valora la importancia de estas interacciones entre las partes del sistema y entre los sistemas entre sí y puede planificar estrategias para intervenir favorablemente dentro y entre sistemas.

Trabajar con un enfoque centrado en la familia significa trabajar fundamentalmente con la unidad familiar como grupo y no tan sólo como la suma de sus miembros. Implica considerar e incluir a la familia en el proceso de prestación de cuidados.

Taylor $^{38}$ entiende a la familia desde tres perspectivas:

1. Como un factor, que junto con otros condicionantes básicos, influye directamente en necesidades de cuidados de sus miembros y la capacidad y posibilidad de estos de satisfacer dichas necesidades. 
2. Como un contexto en el que frecuentemente se proporcionan cuidados a un miembro dependiente. 3. Como una entidad global que puede, en determinadas circunstancias, necesitar cuidados enfermeros transformándose en "una unidad de cuidados".

Desde este punto de vista la familia se considera un centro de interés en sí misma. Es un sistema en la cual se produce una intensa, estrecha y duradera interacción entre los componentes que se pone de manifiesto en materia de autocuidado. La familia constituye una fuente de apoyo social inmediato para sus miembros, aunque debido a los cambios socioculturales que está sufriendo la familia, es necesario ser cuidadoso en este último punto ya que no todas las familias pueden proporcionar un apoyo eficaz a sus miembros.

Es necesario que el profesional conozca el tipo de familia y el ciclo vital porque esto le permitirá relacionar el tipo de familia con el proceso salud/enfermedad, identificar la capacidad de la familia para obtener recursos delante de una crisis, conocer el comportamiento de la familia con relación a la utilización de los servicios sanitarios, planificar actividades curativas, preventivas y de promoción de la salud y utilizar recursos individuales, familiares o comunitarios. Debe realizarse una valoración de las necesidades del grupo familiar teniendo en cuenta las características del grupo familiar, estructura, desarrollo, relaciones; los hábitos de vida y el estado de salud de cada uno de sus miembros; las condiciones de la vivienda; el trasfondo cultural de la familia y sus relaciones con la comunidad.

Los objetivos de atención a la familia son: Proporcionar una atención continuada. Ayudar a la familia a prepararse para eventualidades previsibles. Potenciar y fomentar el autocuidado. Educar para que sean capaces de proporcionar cuidados de calidad. Ayudarles a afrontar las crisis de forma adaptativa, y a identificar, buscar y utilizar recursos de manera adecuada. Además, desarrollar un sentimiento de unidad y promover la cooperación, el apoyo mutuo y la coordinación dentro del grupo familiar.

La salud familiar debe medirse por los siguientes indicadores: La salud individual de cada uno de sus miembros, Si sus roles están bien estructurados y se adaptan a las necesidades cambiantes, si existen patrones de comunicación afectivos, por su capacidad para crear y mantener un hogar y un estilo de vida saludable, por su capacidad para afrontar los problemas y por sus relaciones con la comunidad.

El cuidador principal debe desarrollar multitud de tareas para la persona que recibe cuidados: higiene, alimentación, movilización, curas, compañía, trámites burocráticos, soporte emocional, debe hacer de intermediario y ha de tomar decisiones. Todo ello puede convertirlo en enfermo secundario, ya se han comentado las repercusiones físicas, psicológicas y sociales asociadas al cuidar.

El profesional de enfermería debe posicionarse ante la familia del enfermo tratándola como un recurso importante del sistema sanitario, como un cuidador más, pero también como otro cliente del servicio de salud. Para ello debe establecer relaciones de igualdad y cooperación con el sistema informal de cuidados, ha de utilizar la alianza terapéutica y ha de observar cada uno de los problemas individuales, incluidos los del cuidador, dentro del marco del grupo familiar.

Aunque las necesidades del cuidador se han de evaluar individualmente hay algunos puntos esenciales para ofrecer apoyo a los cuidadores informales:

1. Proporcionar formación sobre cuidados con el objetivo de mejorar la prestación, pero también para reducir el estrés por la inseguridad de no saber prestar cuidados de calidad. Además utilizar una técnica adecuada mejora no sólo el resultado final sino que puede disminuir el esfuerzo requeri- 
do, por ejemplo saber aplicar técnicas de ergonomía para la movilización o aprender técnicas para la higiene del paciente encamado.

2. Proporcionar información sobre planificación de actividades. Como gestionar mejor el tiempo. 3. Enseñar a obtener apoyo de otras personas bien mediante técnicas de asertividad entrenando al cuidador para que sea capaz de identificar otras fuentes de apoyo informal y sea capaz de pedir ayuda y obtener una mayor participación de otros miembros de la familia o informándoles de los sistemas de apoyo formal: servicios sanitarios, sociales o voluntariado.

4. Identificar los problemas de salud de la cuidadora, diagnosticarlos y tratarlos adecuadamente. Ofrecer apoyo emocional y enseñar estrategias de afrontamiento al estrés.

5. Facilitar o favorecer la comunicación cuidador/beneficiario cuando esta esté alterada.

\section{BIBLIOGRAFÍA:}

1. Garcia Calvente M.M.; Mateo Rodriguez I. "El cuidado de la salud. ¿Quién hace qué?" Índex de Enfermería 1993;6:16-19

2. WENGER, GC. "The major English-speaking countries". En Kending H. Hashimoto A. Coppard LC. Editores. Family support for the Elderly. The international experience. Oxford: Oxford University Press. 1992: 117-37

3. Duran MA. "El tiempo y la economía española". ICE. 1991:695: 9-48

4. OCDE. "The reform of health care systems. A review of seventeen OCDE countries". OCDE. Health policy Studies no. 5. París. OCDE 1994

5. INSERSO. Las personas mayores en España. Perfiles. Reciprocidad familiar. Servicios Sociales. Ministerio de Servicios Sociales. 1997

6. Informe SESPAS 1998. "La familia y el Estado del Bienestar" Escuela Andaluza de Salud Pública. 1998

7. Bermejo F; Ribera J; y otros. Grupo NEDiCES. "Aspectos del cuidador sociofamiliar al paciente con demencia. Datos estudio poblacional en dos zonas de Madrid". Revista Gerontológica. 1997;7:92-99

8. ZunZUNEGUI MV; "Envejecimiento y Salud" en Informe SESPAS 1998. "La familia y el Estado del Bienestar. Escuela Andaluza de Salud Pública. 1998
9. TWIGG J. "Cuidadores de los ancianos: modelos para un análisis" en Jamieson A.; Illsley R. (de) Comparación de políticas europeas de atención a las personas mayores. Barcelona: SG editores. 1993.

10. Bazo MT:; Dominguez-Alcón C. "Los cuidados familiares de salud en las personas ancianas y las políticas sanitarias" en Revista española de investigaciones sociológicas. N. ${ }^{\circ}$ monográfico sobre envejecimiento. Noviembre, 1995: 4-22.

11. JAmiESON A. "Atención informal en Europa" en Jamieson A.; Illesley R. ed. Comparación de políticas europeas de atención a las personas ancianas. Barcelona: SG editores, 1993.

12. RoBinson $K$. "The relationships between social skill, social support, self-steem and burden in adult caregivers" Advan Nursing 1990;15:788-795.

13. Cuesta de La C. "Familia y salud" Rev, Rol de Enfermería 1995;203:21-24

14. Pérez JM.; Alberto J.; LabarTa S. "El síndrome del cuidador en los procesos con deterioro cognitivo" Atención Primaria 1996; 18:82-98.

15. Ashi S.; Girona G.; Ortuño MA. "Síndrome del cuidador" Rehabilitación 1995; 29:465-468.

16. GARCIA L.; PARDO C. "Factores asociados a la ansiedad y toma de psicofármacos en cuidadores de pacientes incapacitados" Atención Primaria 1996;18:103-110.

17. NOLEN MR. GLRANT G. Adressing the needs of informal carers: a negleter area of nursing practice" Advan Nursing 1984;14:950-961.

18. Segura M.; Bastida N.; Martí N.; Riba M. "Los enfermos crónicos domiciliarios y su repercusión en los cuidadores principales" Atención Primaria 1998; 21 (7): 431-436.

19. Rubio ML.; Sánchez A.; Ibáñez JA; Galbe J.; Martó N.; Mariscal J; ; "Perfil medicosocial del cuidador principal. ¿Se trata de un paciente oculto?". Atención Primaria 1995;16 (4):181-186.

20. Puig C.; Hernández lM.; Gervás JJ. "Los enfermos crónicos recluidos en el domicilio y su repercusión en la familia: Una investigación cualitativa" Atención Primaria 1992; 10 (1): 550-552.

21. Bilbao I.; Gastaminza AM.; Garcoa JA.; Quindimil JA.; LÓPEZ JL.; Huidobro L. "Los pacientes domiciliarios crónicos y su entorno en atención primaria de salud" Atención Primaria 1994; 13 (4): 188-190.

22. Martin M.; Salvado I.; Nadal S.; Mij LC.; Rico JM.; Lanz P; TAussig ML. "Adaptación para nuestro medio de la escala de sobrecarga para el cuidador de Zarit. Rev. Gerontológica 1996; 6 (4):338-346.

23. Roca M.; Úbeda I.; Garcia L.; Fuentalsaz C.; Verdura T. ¿Se cuida a la familia de los enfermos crónicos? Rev. Rol de Enfermería 1999; 22 (1): 75-78.

24. Sorrentino AM. "La familia frente a la deficiencia física" en Handicap y rehabilitación. Una brújula sistémica en el uni- 
verso relacional del niño con deficiencias físicas. Ed. Paidós 1990: 46 .

25. Silberstein Goldenberg D. "La familia, un paciente olvidado" Rev. Rol de Enfermería 1997; 223: 25-30.

26. ÚBEDA I; PUJOL G. "La familia como unidad de atención en salud comunitaria" en Caja C.; López RM. Enfermería Comunitaria II. Ed. Masson 1993; (8): 87-93.

27. LEININGER MM. "Transcultural care diversity and universaly: a theory of nurs". Nurs health care. 1985;6(4):209-212.

28. ColliĖe MF. "Promover a vida" Trad. Braga ML. Lisboa. Printipo-Damala 1989

29. PARSONS T.; BAles R. "Family, socialitation and interaction process" Glencoe III, Free Press, 1955.

30. Fernández A.; FernANDEZ MP. "Intervención de la enfermera de asistencia domiciliaria en el sistema familiar" Enfermería Clínica 1995; 5 (5): 216-220.

31. Ruiz M.; Muñoz MD.; Maza MA.; Martinez MN. "Educación para la salud a cuidadores informales" Enfermería Clinica 1998; 8 (4): 171-177.

32. Rivera J.; Bermejo F; Trincado R.; Morales JM.; "Aspectos del cuidado sociofamiliar al paciente con demencia.
Aspectos específicos del cuidado familiar" Rev. Gerontológica 1997; 7 (3): 138-147.

33. Amezcúa M.; Arroyo MC.; Montes N.; López E.; Pinto I.; Cobos F. " Cuidadores familiares: Su influencia sobre la autonomia del paciente incapacitado" Enfermería Clínica $1996 ; 6(6): 233-241$.

34. Úbeda I.; Roca M.; Garcia L. "Presente y futuro de los cuidados informales" Enfermería Clínica 1996; 8 (3): 116-121.

35. Perlado F. "Apoyo a los cuidadores de enfermos de Alzheimer" Rev. Gerontológica 1995; monográfico: 47-53.

36. Montoro J.; Kosloski K.; Satre J.; Colmenero E. "Los cuidadores familiares y el uso de servicios asistenciales" Rev. Gerontológica 1996; 6: 174-180.

37. Alfaro R. "Aplicación del proceso de Enfermería" ed. Doyma Barcelona. 1988.

38. TYLOR S. "An interpretation of family within Oren's General Theory of Nurs". Nurs science Quarterly, 1989; 2:131-137.

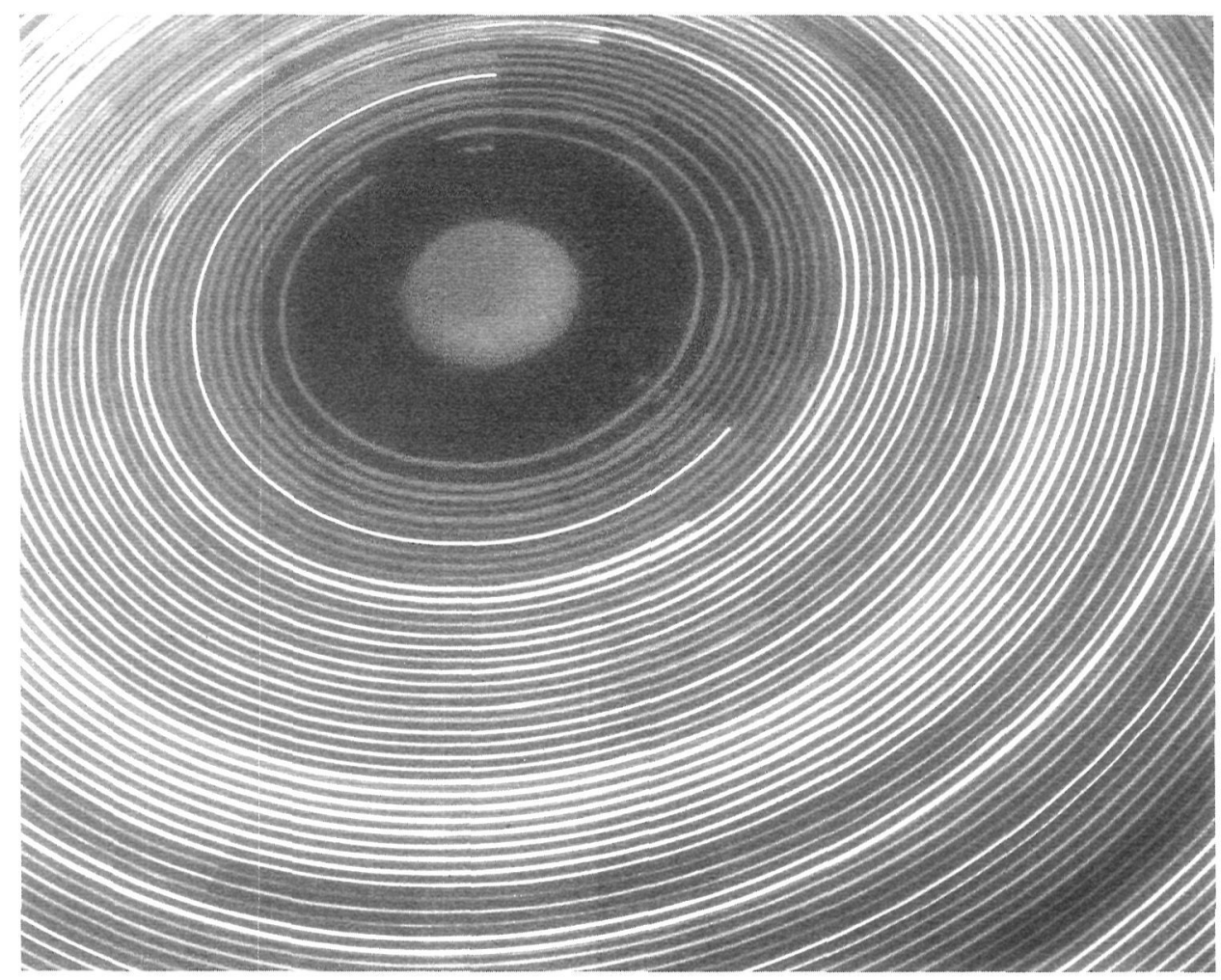

